\title{
NSVS 01031772 Cam: A New Low-Mass Triple?
}

\author{
M. Wolf ${ }^{1}$, P. Zasche ${ }^{1}$, K. Hornoch ${ }^{2}$, M. Chrastina ${ }^{3}$, J. Janík ${ }^{3}$, \\ and M. Zejda ${ }^{3}$ \\ ${ }^{1}$ Astronomical Institute, Faculty of Mathematics and Physics, Charles University Prague, \\ CZ-180 00 Praha 8, V Holešovičkách 2, Czech Republic, email: wolf@cesnet.cz \\ ${ }^{2}$ Astronomical Institute, Academy of Sciences, CZ-251 65 Ondřejov, Czech Republic \\ ${ }^{3}$ Institute of Theoretical Physics and Astrophysics, Masaryk University, Brno, Czech Republic
}

Abstract. We present a photometric study of the newly discovered low-mass eclipsing binary NSVS 01031772 Cam based on observations obtained at Ondřejov observatory from $2007-2011$.

Most determinations of the fundamental parameters of low-mass stars using eclipsing binaries indicate a strong discrepancy between theory and observations. The measurements clearly indicate that the observed radii are generally larger than the predictions by stellar models. The eclipsing binary NSVS 01031772 Cam (GSC 4561.647, V=12.6 mag) was discovered by McIntyre \& Shaw (2005) as a low-mass, double-lined and detached eclipsing binary in the Northern Sky Variability Survey (NSVS). The comprehensive study was later presented by López-Morales et al. (2006, hereafter LM06), who found the precise masses of both components and discovered that the radius of each component exceeds the evolutionary model by about $8.5 \%$ on average. In this paper, we present improved system parameters based on our new photometric observations.

Our new CCD photometry was obtained at Ondřejov Observatory, Czech Republic, during 2007-2011. We used mostly the CCD camera G2-3200 of Moravian Instruments attached to the $0.65-\mathrm{m}$ telescope, and $V R$ photometric filters. We used the same comparison star GSC 4561.0787 as done by previous investigators. The processing of CCD frame series was done routinely by the Aрнот software package. The supplementary CCD photometry was obtained at Masaryk University Observatory, Brno, during 20092011. The 0.62-m reflecting telescope, the CCD camera SBIG ST-8, and $V R$ filters were used. The additional measurements were secured by JJ during one night at Mt. Suhora Observatory, Poland, using the 0.65-m telescope and BVR filters in November 2009.

The new times of mid-eclipse were determined using the profile fitting method; the complete list will be presented elsewhere. Using published minima and our new timings, we improved the linear light elements: Pri.Min. = HJD 24 53456.68023(8) + 0.36814052(6)E.

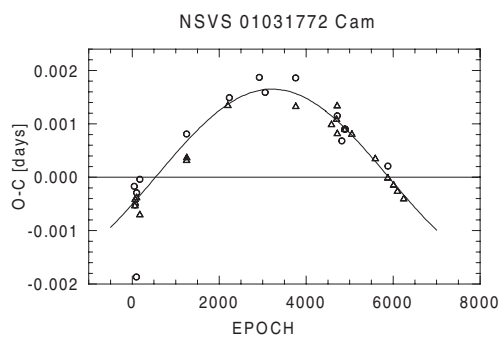

Figure 1. The $O-C$ diagram of NSVS 01031772. The primary and secondary minima are denoted by circles and triangles. The curve denotes the possible LITE with the 11-yr period. 

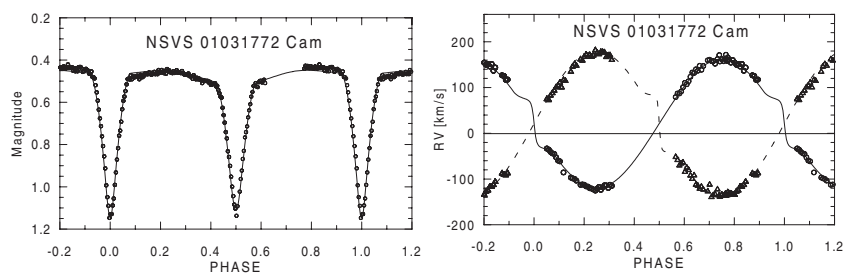

Figure 2. Left panel: The Phoевe solution of the $V$ light curve obtained at Ondřejov with one spot on the primary component $\left(T_{f}=0.95\right.$, latitude $90^{\circ}$, longitude $180^{\circ}$ and radius $\left.25^{\circ}\right)$. Right panel: The RV curve solution. Circles for primary, triangles for secondary RVs.

Table 1. Physical parameters of NSVS 01031772 Cam.

\begin{tabular}{lccc} 
Element & this paper & McIntyre \& Shaw $(2005)$ & LM06 \\
\hline$P$ [day] & $0.36814052(6)$ & 0.368154 & 0.368141 (fixed) \\
$i$ [deg] & $85.7 \pm 0.1$ & $88 \pm 0.5$ & $85.91 \pm 0.03$ \\
$M_{1}\left[\mathrm{M}_{\odot}\right]$ & $0.555 \pm 0.005$ & - & $0.543 \pm 0.003$ \\
$M_{2}\left[\mathrm{M}_{\odot}\right]$ & $0.495 \pm 0.005$ & - & $0.498 \pm 0.003$ \\
$T_{1}[\mathrm{~K}]$ & 3500 (fixed) & $2885 \pm 5$ & $3615 \pm 72$ \\
$T_{2}[\mathrm{~K}]$ & $3455 \pm 25$ & $2895 \pm 5$ & $3513 \pm 31$ \\
$R_{1}\left[\mathrm{R}_{\odot}\right]$ & $0.592 \pm 0.005$ & - & $0.526 \pm 0.003$ \\
$R_{2}\left[\mathrm{R}_{\odot}\right]$ & $0.492 \pm 0.005$ & - & $0.509 \pm 0.003$ \\
\hline
\end{tabular}

A significant sinusoidal period change is clearly visible on the current $O-C$ diagram (see Fig. 1). We tried to fit the $O-C$ values by a light-time effect (hereafter LITE) caused by the orbiting third body. The preliminary solution of the third body's circular orbit gives a minimal period of about 3900 days (11 years) and a semi-amplitude of about 0.00165 days $(2.4 \mathrm{~min})$. The minimal mass of the third body in a coplanar orbit follows as $0.063 \mathrm{M}_{\odot}$ or 65 Jupiter mass only.

Our own $V R$ light curves and radial velocity curves of LM06 were reduced using the current PhoEBe computer code, version 31a, developed by Prša \& Zwitter (2005). The gravity darkening exponents were set 0.045 according to LM06. The bolometric albedo $A=0.5$ corresponding to stars with a convective envelope was taken for each star. Since the light curve of the system has a wave-like distortion, we included spots in our solution, which are parameterised in the same way as in the Wilson and Devinney code: the temperature factor $T_{f}$, the latitude and longitude of the spot centre, and the angular radius of the spot. The resulting elements given in Table 2 are compared with the previous solutions of McIntyre \& Shaw (2005) and LM06.

Adopting masses of both components given in Table 2, the radii according to theoretical models of Baraffe et al. (1998; $Z=0.02,0.35$ Gyr isochrone) should be about 0.50 and $0.46 \mathrm{R}_{\odot}$, which are lower than our results. The current spot activity could be well reproduced by one spot on the primary component. New high-accuracy timings of this low-mass eclipsing binary are necessary in the near future to improve the LITE parameters derived in this paper and to confirm the 11-year orbital period of the possible third body.

This research was supported by grants GAČR 205/08/H005 and P205/10/0715; Research Program MSM0021620860 of the Ministry of Education (to MW, PZ); and GAAV IAA301630901, MEB051018 and MUNI/A/0968/2009 (to MCh, JJ, MZ). We thank Dr. M. López-Morales for the use of their original data.

\section{References}

Baraffe, I., Chabrier, G., Allard, F., \& Hauschildt, P. H. 1998, A\&A 337, 403

López-Morales, M., Orosz, J. A., Staw, J. S., Havelka, L. et al., 2006, astro-ph/0610225v1, LM06 McIntyre, T. \& Shaw, J. S. 2005, IAPPP Comm. 101, 38

Prša, A. \& Zwitter T. 2005, ApJ 628, 426 